\title{
El TC687 "Creación de espacios de fortalecimiento de habilidades para la vida que prevengan el consumo de drogas en la adolescencia y la juventud"de la Universidad de Costa Rica (2018-2019): un análisis desde la metodología de la sistematización de experiencias
}

\section{"Creating spaces to strengthen life skills preventing drug use in adolescence and youth" (a project run by the University of Costa Rica, 2018-2019): An analysis of the TC687 from the methodology of the systematization of experiences}

Daniel Martínez Esquivel

Universidad de Costa Rica

San José, Costa Rica daniel.martinezesquivel@ucr.ac.cr

Recibido: 2/09/2019 Aceptado:29/11/2019

Resumen. En esta investigación se reconstruye la experiencia del trabajo comunal de la Universidad de Costa Rica TC687 sobre el fortalecimiento de habilidades para la vida realizado durante el período 2018-2019, con el objetivo de fortalecer la participación social del estudiantado activo en trabajo comunal universitario que contribuya a la satisfacción de las necesidades de las comunidades de Costa Rica, a partir de la sistematización de experiencias de la propuesta de Óscar Jara (2012) como estrategia metodológica. Para la recolección de la información se utilizó la ficha de recuperación de aprendizajes, cuyo resultado principal es la participación estudiantil, la cual se fundamentó en la capacitación previa para realizar trabajo de campo, además del diagnóstico, de la planeación, la ejecución, la evaluación y el registro de estrategias 
Revista Universidad en DiÁlogo • Vol. 10, N. ${ }^{\circ}$ 1, Enero-Junio, 2020 • 145-161

ISSN 2215-2849 • EISSN: 2215-4752

URL: http://www.revistas.una.ac.cr/index.php/dialogo/index CorReo ElECTRÓNICO: universidadendialogo@una.cr

DOI: https://doi.org/10.15359/udre.10-1.9

educativas con enfoque de habilidades para la vida que prevengan el consumo de drogas en poblaciones en riesgo. El TC687 fortalece la participación de la población estudiantil al capacitarla para que tenga un acercamiento a diferentes realidades que la sensibilizan con las personas y sus necesidades. Además, a partir de la experiencia, tanto el estudiantado como la comunidad, fortalecen las habilidades para la vida.

Palabras clave: adaptación, participación comunitaria, prevención de enfermedades, trastornos relacionados con sustancias.

\begin{abstract}
This research reconstructs the experience of the social work project \#TC687 to strengthen life skills, run by the University of Costa Rica from 2018 to 2019. To strengthen the social participation of university students that contribute to meeting the needs of Costa Rican communities. Systematization of experiences based on the proposal by Óscar Jara (2012) as a methodological strategy. To collect the information, learning recovery sheets were used. Students' participation was based on prior training to perform fieldwork, besides the diagnosis, planning, execution, evaluation, and registration of educational strategies with a focus on life skills that prevent drug use in populations at risk. The TC687 project strengthens student participation by bringing it closer to different realities that sensitize it to people and their needs. In addition, from experience, both students and the community strengthen life skills.
\end{abstract}

Keywords: adaptation, community participation, disease prevention, substancerelated disorders.

\title{
Introducción
}

El presente artículo es una sistematización de la experiencia del trabajo comunal universitario (TCU) "Creación de espacios de fortalecimiento de habilidades para la vida que prevengan el consumo de drogas en la adolescencia y la juventud" (TC687), en el proceso y dinamismo que tiene como proyecto adscrito a la Vicerrectoría de Acción Social de la Universidad de Costa Rica -en adelante UCR-. Dicha sistematización se refiere al período de vigencia desde el 1 de marzo de 2018 hasta el 29 de febrero de 2019.

Por un lado, el TCU es una actividad de acción social que tiene como propósito establecer una relación redituable entre grupos-comunidades vulnerables y el estudiantado de la UCR para contribuir en las transformaciones sociales a partir de la satisfacción de las necesidades más importantes (UCR, 2018). En el caso del TC687, este se caracteriza por la creación de espacios para la implementación de estrategias educativas y lúdicas con un enfoque de habilidades para la vida -en adelante $\mathrm{HpV}$ - en poblaciones de adolescentes y de jóvenes que mantienen riesgos relacionados con el consumo de drogas. 
URL: http://www.revistas.una.ac.cr/index.php/dialogo/index

CORREO ELECTRÓNICO: universidadendialogo@una.cr

DOI: https://doi.org/10.15359/udre.10-1.9

Las $\mathrm{HpV}$ se pueden definir como un conjunto de destrezas que les permite enfrentarse de la mejor manera a los desafíos cotidianos con una actitud positiva hacia el bienestar (Díaz y Mejía, 2018). Existe variabilidad en la clasificación de las HpV, Mantilla (2001) identifica diez principales: conocimiento de sí mismo, comunicación efectiva, toma de decisiones, pensamiento creativo, manejo de sentimientos y emociones, empatía, relaciones interpersonales, solución de problemas y conflictos, pensamiento crítico y manejo del estrés. Además, la Organización Panamericana de la Salud (OPS, 2001) categoriza las HpV como habilidades sociales, habilidades cognitivas y habilidades para el control de las emociones.

Sin embargo, ambas coinciden en que las $\mathrm{HpV}$ deben emplearse por medio de una metodología interactiva donde el facilitador y el participante tengan la posibilidad de adoptar una actitud que les permita construir conocimientos según su propia experiencia de manera lúdica en un ambiente seguro (Mantilla, 2001; OPS, 2001).

En este sentido, ambas perspectivas son pertinentes para el cumplimiento de los objetivos del TC687, entretanto se reconozca que las habilidades no se emplean de forma individualizada, por el contrario, cada una complementa a la otra para que en conjunto se refuercen. Es importante mencionar que las $\mathrm{HpV}$ se han empleado en el currículo y en los diferentes programas tanto a nivel internacional como nacional dirigidos a la prevención del consumo y a fortalecer el bienestar de las personas en general.

A nivel internacional, García et al. (2017) implementaron un programa basado en $\mathrm{HpV}$ en una comunidad mexicana que vive en extrema pobreza para mejorar sus estrategias de afrontamiento. Los resultados evidenciaron efectos positivos en las conductas adoptadas hacia el mejoramiento de su salud. Asimismo, Corrales, Quijano y Góngora (2017) presentaron los resultados de un programa de intervención de $\mathrm{HpV}$ en adolescentes mexicanos de un centro de enseñanza, observando una reducción de algunas conductas inadecuadas y un incremento de algunas adecuadas.

Singla et al. (2019) desarrollaron una revisión sistémica y un metaanálisis para identificar la implementación y la efectividad de programas sobre $\mathrm{HpV}$ en adolescentes en países de mediano y bajo ingreso, demostrando sus beneficios en la reducción de riesgos en áreas como la salud mental. Por último, Díaz y Mejía (2018) realizaron una evaluación del modelo de $\mathrm{HpV}$ propuesto por la Organización Mundial de la Salud (OMS) y la OPS en tres instituciones educativas colombianas, concluyendo que la estrategia debe trascender la enseñanza de los conceptos para convertirse en un eje central de los procesos formativos. 
Revista Universidad en DiÁlogo • Vol. 10, N. ${ }^{\circ}$ 1, Enero-Junio, 2020 • 145-161

ISSN 2215-2849 • EISSN: 2215-4752

URL: http://www.revistas.una.ac.cr/index.php/dialogo/index CORREO ELECTRÓNICO: universidadendialogo@una.cr

DOI: https://doi.org/10.15359/udre.10-1.9

A nivel nacional, el entrenamiento de las $\mathrm{HpV}$ forma parte del plan de estudios de la Licenciatura de Enfermería de la UCR, lo que ha generado diferentes trabajos finales de graduación. El caso de Murillo y Ramírez (2014), quienes ejecutaron un programa de enfermería para el desarrollo de $\mathrm{HpV}$ en niños y en adolescentes de la comunidad de Birrisito en Heredia. Esta investigación evidencia que es necesario desarrollar procesos a largo plazo para el entrenamiento de HpV. Recientemente, Badilla y Salazar (2019) implementaron una intervención de enfermería para el fortalecimiento de $\mathrm{HpV}$ en niños de la provincia de Limón. Comparando la valoración inicial con la final, registraron un mejoramiento en las habilidades sociales, cognitivas y emocionales.

La evidencia presentada apoya el desarrollo de estrategias de $\mathrm{HpV}$ y representan estrategias que se podrían seguir o replicar. Por consiguiente, justifica la importancia de plasmar su experiencia a través de este estudio para su divulgación.

Por otro lado, de acuerdo con Jara (2012), la sistematización de experiencias se puede definir como un proceso reflexivo, ya sea individual o colectivo, en torno a una experiencia realizada o vivida, de carácter sociohistórico y a partir de la cual se hace una reconstrucción ordenada de lo ocurrido para producir nuevos conocimientos desde una mirada crítica de la práctica. En este sentido, la sistematización de esta actividad de acción social permite la construcción de evidencias que fortalecen la importancia que tiene para la sociedad. Incluso, es un respaldo que justifica su relevancia ante la UCR, las unidades académicas y las personas que no están familiarizadas con su dinámica.

Menciona Jara (2012) que la sistematización inicia con el punto de partida que se refiere a la experiencia. En el presente artículo la experiencia es el TCU en sí mismo, en específico el TC687. Seguidamente, se formula un plan de sistematización a partir del cual se derivan diversas interrogantes cuyas respuestas definen lo analizado en el presente documento (Jara, 2012). Dichas preguntas se presentan a continuación:

- ¿Para qué queremos sistematizar? La respuesta se refiere al objetivo del presente artículo, el cual es fortalecer la participación social del estudiantado activo en un trabajo comunal universitario que contribuya a la satisfacción de las necesidades de las comunidades de Costa Rica a partir de la sistematización de experiencias.

- ¿Qué experiencias queremos sistematizar? Son las experiencias en sí mismas, el TC687 en su totalidad, refiriéndose a todas las actividades que implican su desarrollo para el cumplimiento de los objetivos y de las metas. 
- ¿Qué aspectos centrales nos interesan más? Al definir el eje de esta sistematización, se pensó en la participación docente-estudiantil y sus implicaciones en la participación durante el TCU.

\section{Material y método}

Se utilizó la propuesta metodológica de Jara (2012) para la sistematización de experiencias, la cual consta de cinco tiempos que se anuncian a continuación:

- El punto de partida: la experiencia. Se parte de la propia experiencia tomando en cuenta dos requisitos, haber participado y contar con registros. En el caso del TC687, cada estudiante cuenta con un plan de trabajo y con un instrumento de registro que plasma en el informe final de cada actividad en la que participa.

- Formular un plan de sistematización. Se inicia el proceso de sistematización a partir de la definición del objetivo, del objeto y del eje.

- La recuperación del proceso vivido. Es un ejercicio descriptivo y narrativo del trayecto seguido por las experiencias.

- Las reflexiones de fondo. Es un proceso de análisis y síntesis sobre el proceso vivido y desde la riqueza de la propia experiencia.

- Los puntos de llegada. Se formulan conclusiones y aprendizajes que permitan mejorar la práctica.

El contexto de esta sistematización se desarrolló principalmente en la UCR, ya que fue donde se realizaron las actividades de capacitación, coordinación y planeación con el profesorado y el estudiantado. Sin embargo, se incluyeron experiencias de campo dadas en diferentes instituciones, como el Área Rectora de Salud Curridabat, el Patronato Nacional de la Infancia (PANI), el Colegio Nocturno La Unión, el Pueblito y el Instituto sobre Alcoholismo y Farmacodependencia (IAFA). Los participantes fueron los profesores responsable y colaborador, y el estudiantado activo en el TCU durante el período señalado.

Los participantes fueron cuarenta y cinco estudiantes activos del TCU de diferentes áreas como enfermería, derecho, educación especial, medicina, psicología, orientación, ingeniería eléctrica, ingeniería civil, promoción de la salud, estadística y farmacia; además de los profesores coordinador y colaborador. 
Además, para la recolección de la información se utilizó la "Ficha de recuperación de aprendizajes" propuesta por Jara (2012). Este instrumento se divide en cuatro temas que guían el registro de la información: el contexto de la situación, el relato de lo ocurrido, los aprendizajes y las recomendaciones.

\section{Consideraciones éticas}

E1 TC687 es un proyecto adscrito a la Vicerrectoría de Acción Social (VAS) de la UCR con el número de inscripción TC-687. Este se rige por el "Reglamento del Trabajo Comunal Universitario de la Universidad de Costa Rica", normativa encargada de la regulación y la organización de su funcionamiento para la garantía de sus buenas prácticas en las relaciones docente-estudiantadocomunidad. Además, esta actividad responde a los principios y los propósitos que se establecen en el "Estatuto Orgánico de la Universidad de Costa Rica" que se reafirman en la ética y los derechos humanos (UCR, 2018; UCR, 1974).

\section{Resultados}

Los resultados obtenidos se derivan de la experiencia misma de la cual el autor ha formado parte. Corresponde a la recuperación del proceso vivido de esta actividad de acción social según el período indicado de manera narrativa y descriptiva (Jara, 2012).

\section{Contexto de la situación}

E1 TC687 es una actividad de acción social de la Escuela de Enfermería, por lo que físicamente se ubica en esta unidad académica, lugar donde se desarrollaron las reuniones informativas, de orientación, de inducción y planeación. Tales se sustentaron en la participación estudiantil. En la UCR cada estudiante tiene la oportunidad de matricular su TCU durante los meses de marzo, agosto y noviembre, donde adquiere el compromiso de cumplir con trescientas horas de trabajo de campo, para el estudiantado de grado.

En esta oportunidad, colaboraron en el proceso alrededor de cuarenta y cinco estudiantes de diferentes áreas como enfermería, derecho, educación especial, medicina, psicología, orientación, ingeniería eléctrica, ingeniería civil, promoción de la salud, estadística y farmacia. Esto permitió conformar un equipo de trabajo interdisciplinario y multidisciplinario, ya que cada carrera aportó de sus conocimientos, interactuando entre sí con el propósito de prevenir el consumo de sustancias a partir del enfoque de $\mathrm{HpV}$ en poblaciones de adolescentes y de jóvenes. 
URL: http://www.revistas.una.ac.cr/index.php/dialogo/index

CORREO ELECTRÓNICO: universidadendialogo@una.cr

DOI: https://doi.org/10.15359/udre.10-1.9

Léase la siguiente opinión: "Ha sido ameno, no me he sentido estresado. He hecho muy buen equipo de trabajo" (Estudiante 1, TCU). En el relato se manifiesta el ambiente positivo en el que se desarrolla el proyecto y la importancia del trabajo en equipo.

Cabe destacar que la oferta incluyó actividades de lunes a sábado en horarios de la mañana, de la tarde y de la noche en ubicaciones geográficas como Tirrases de Curridabat, cantón Central de San José, San Diego de La Unión, Paraíso de Cartago y San Pedro de Montes de Oca. Ante lo cual un estudiante mencionó: "Nos dan libertad, no se sigue una estructura, sino que podemos elegir las actividades para el desarrollo del TCU" (Estudiante 3, TCU), ejemplificando el dinamismo con el que se rige el estudiantado para participar de todas las oportunidades de acción en comunidad.

\section{Relato de lo ocurrido}

La dinámica del TCU implicó que la conformación de cada grupo de estudiantes matriculados nuevos se sometiera a un período de orientación e inducción de corte holístico, en función de las actividades que implementaron en la comunidad, con el fin de generar conocimientos a partir de la vivencia de cada uno. Este período estuvo a cargo de estudiantes regulares del TCU bajo la supervisión de los profesores responsable y colaborador. Ello también incluyó estrategias didácticas bajo la modalidad de taller, como la lluvia de ideas, la presentación oral, los esquemas, las actividades lúdicas, entre otras. Se eligieron ambientes internos como aulas para su desarrollo, pero también se aprovecharon los espacios al aire libre disponibles en la institución. Al respecto, se mencionó: "En la inducción nos dio chance de conocernos y eso facilita el trabajo"(Estudiante 3, TCU). En el relato se resalta la importancia que tiene la inducción para la interacción entre el estudiantado y la conformación del grupo.

Además de prepararse para el trabajo de campo, se buscó crear una relación interpersonal de ayuda docentes-estudiantes. También, de manera introspectiva, que el grupo pusiera en práctica cada habilidad para la vida en su cotidiano. Por lo tanto, se dirigió sobre dos vertientes: la población estudiantil matriculada y las poblaciones vulnerables, promoviendo la estrategia ganar-ganar, la cual consiste en obtener beneficios mutuos.

Algunos estudiantes consideraron lo siguiente: "Estos temas nos ayudan a hacer el análisis introspectivo, a veces me pregunto: ¿qué estoy haciendo yo? En lo personal me ha ayudado" (Estudiante 2, TCU). "Lo que más he aprendido es la comunicación y la relación con otras personas porque en mi carrera son solo 
números" (Estudiante 1, TCU). Los relatos ponen en evidencia el impacto que tiene el desarrollo de las habilidades para la vida en el estudiantado, ya que al ponerlas en práctica encuentran beneficios para su cotidianeidad y les facilita el trabajo en comunidad a partir de su propia experiencia.

Adquiridas las competencias necesarias, se elaboró un plan de trabajo con cada estudiante en función de sus posibilidades, asegurando su compromiso con esa actividad sustantiva y sus deberes académicos.

En cada comunidad, los profesores responsable y colaborador coordinaron con diferentes autoridades la posibilidad de que se autorizara la ejecución del proyecto en función del cumplimiento de sus objetivos y metas. Las instituciones donde el TCU contribuyó fueron:

- Área Rectora de Salud Curridabat

- Oficina Local San José Oeste del PANI

- Colegio Nocturno La Unión

- Pueblito

- IAFA

Las actividades desarrolladas gozaron de diversidad, sin embargo, se centraron en la implementación de estrategias educativas sobre las $\mathrm{HpV}$ y la prevención del consumo de drogas. Es importante señalar que previo a esta ejecución, se elaboró un diagnóstico en el que participaron las personas encargadas de cada institución a partir de sus conocimientos sobre la situación a la que se enfrentaría el estudiantado.

Con el diagnóstico claro, se planearon las actividades a trabajar. Durante el proceso de planeación, el estudiantado empleó sus conocimientos disciplinares y tuvo acceso a elementos teóricos de apoyo, como informes sobre las $\mathrm{HpV}$, programas de las $\mathrm{HpV}$, artículos científicos, entre otros, documentos importantes debido a que brindaron los conocimientos necesarios y en ocasiones permitió la replicación de las actividades. Además, hizo uso de la creatividad y de la innovación para elaborar las estrategias y construir materiales aptos para cada población.

Así el caso del Área Rectora de Salud de Curridabat, donde se planeó una actividad de cinco sesiones para trabajar las $\mathrm{HpV}$ agrupadas en sociales, cognitivas y emocionales, y la prevención del consumo de sustancias en un grupo de adolescentes de Tirrases; en cuanto a la Oficina Local San José Oeste del PANI, se participó en el proyecto Promotores de Espacios de Convivencia Positiva; y en 
el Colegio Nocturno La Unión se planearon actividades quincenales por cada $\mathrm{HpV}$ dirigidas a estudiantes de séptimo y octavo año de secundaria.

En cuanto a Pueblito, el estudiantado elaboró un programa de las $\mathrm{HpV}$ que se desarrolló durante cinco semanas, cada sábado. Y, por último, en el IAFA la participación estudiantil se ha centrado en el Centro Nacional de Atención Integral en Drogas para personas menores de edad. Léase lo manifestado: "En las actividades los ponemos a trabajar y luego les preguntamos ¿qué entendieron? ¿Cómo se sintieron? Y a partir de ahí explicamos las HpV y cómo ponerlas en práctica" (Estudiante 4, TCU).

...yo me siento muy feliz porque estoy dando algo. Tal vez hoy les dijeron una cualidad positiva que nunca les dicen y que ellos dijeran algo positivo de sí mismos, que por cierto les resulta complicado. Comentarios de deseos de superarse. Eso es muy motivante para lo que uno hace... (Estudiante 7, TCU)

De acuerdo a lo expresado por el estudiantado, se puede observar la dinámica que tiene el TCU en el trabajo de campo en cuanto a su ejecución y la comprobación sobre la transmisión de la información, así como el impacto emocional de las actividades.

Durante la implementación, se enfrentó a grupos con una dinámica compleja, lo que les exigió mayor compromiso para alcanzar su cometido. Fue una oportunidad de acercarse a la realidad de la sociedad costarricense, saliendo de su zona de confort para adquirir la sensibilidad necesaria al ofrecer un trabajo de calidad. En este momento se compartió la duplicidad en las sensaciones y emociones que genera el TCU, permitiéndoles evaluar su proyecto de vida e influir en el de otros. Algunos estudiantes refirieron lo siguiente: "Realmente el TCU ha cambiado mi perspectiva acerca de muchas cosas y particularmente en relación con los jóvenes y el impacto que podemos tener en ellos" (Estudiante 4, TCU). "Conocer la realidad de muchas personas ha sido chocante, pero me ha dejado valorar las oportunidades de mi vida" (Estudiante 2, TCU). "Me llama la atención las personas que han vivido de todo e intentan salir adelante. Uno intenta animarlos, decirles que van bien" (Estudiante 1, TCU).

Los relatos anteriores reflejan la realidad social en la que se desenvuelve el TCU y cómo los estudiantes universitarios identifican condiciones de vida diferentes a las propias, que parecen no ser tan favorables. La confrontación de estas favorece la sensibilidad y el vínculo para desarrollar acciones comprometidas y responsables. 
Al término de cada actividad programada, el estudiantado tuvo la obligación de elaborar una evaluación con la población participante en función de las actividades desarrolladas. Esto le permitió reconocer los alcances logrados, así como tomar las medidas correctivas necesarias para mejorar su práctica en otras instituciones o comunidades.

Para finalizar, cada estudiante elaboró un informe en el que debió incluir información sobre el lugar, la población meta, el cronograma de trabajo, la descripción de las actividades, su experiencia, las limitaciones encontradas y las horas invertidas.

\section{Aprendizajes}

La estrategia de $\mathrm{HpV}$ debe ser una práctica constante y continua en la vida de cada persona. Solo de esta manera se obtendrán resultados positivos a través de la mediación persona a persona. Un estudiante manifestó lo siguiente:

Me llevo muchas enseñanzas, de antemano quiero agradecerle por su compromiso, no solo con buscar nuestro bienestar como estudiantes sino por querer siempre que el TCU efectivamente deje huella en aquellos que tengan contacto con él; todo eso me lo llevo para mi futuro profesional, académico y personal. (Estudiante 9, TCU)

La persona que generó el relato anterior resumió la idea general del TCU, el cual busca impactar de primera mano en los estudiantes activos para que puedan impactar subsiguientemente en las comunidades.

\section{Recomendaciones}

Se considera importante la actualización permanente de las actividades y de las estrategias utilizadas para la adquisición de las $\mathrm{HpV}$, así como mayor contacto con la población. Léase lo que la evidencia señala: "Sería buena una reunión por mes para definir las actividades a realizar o mejorar las matrices que ya existen" (Estudiante 1, TCU). "Me gustaría interactuar más con la población" (Estudiante 2, TCU).

\section{Discusión}

La presente discusión se desarrolla en el marco de las reflexiones de fondo (Jara, 2012). Se basa en el análisis que el autor realiza desde las dimensiones del conocimiento de la enfermería en salud mental, por lo que se integran 
URL: http://www.revistas.una.ac.cr/index.php/dialogo/index

CORREO ELECTRÓNICO: universidadendialogo@una.cr

DOI: https://doi.org/10.15359/udre.10-1.9

diferentes propuestas teóricas de la disciplina para comprender la experiencia misma del TCU como la teoría de las relaciones interpersonales en enfermería, la teoría sobre cuidado, sabiduría clínica y ética en la práctica de enfermería, el modelo de sistemas de Neuman, el modelo de adquisición de competencias, posturas sobre el desarrollo humano y otras.

El TC687 se erige a partir de la interacción entre los profesores, el estudiantado y la comunidad, mediante la adquisición de conocimientos y de habilidades que permitan satisfacer las necesidades imperantes en la sociedad. Respecto a la prevención del consumo de drogas, el TCU está diseñado para ofrecer estrategias universales y selectivas, lo que significa que estas están dirigidas a la población en general, pero también para aquellos grupos que se consideran vulnerables. Sin embargo, no se puede ignorar la complejidad de la dinámica social actual, existiendo muchos casos de personas que ya consumen drogas. Lo anterior hace necesario que el TCU se apoye en profesionales e instituciones que intervengan esta necesidad a través de la prevención indicada (NIDA, 2004), debido a que su abordaje no es uno de los objetivos de este.

Por consiguiente, las relaciones interpersonales son un pilar clave para quienes participan en este proyecto. Peplau (1990) menciona que el establecimiento de una relación interpersonal es un acto terapéutico donde las personas que participan se benefician logrando satisfacer sus necesidades. Estas relaciones terapéuticas son dinámicas y complejas. Requieren un compromiso real para que funcionen en un sentido de ayuda que implica el desarrollo del autoconocimiento, evitando la creación de prejuicios y estableciendo la empatía con las poblaciones meta. Lo anterior favorece el desarrollo de la personalidad y de la adquisición de las habilidades cognitivas, emocionales y sociales (Rogers, 2011), siempre y cuando exista en el facilitador y en el participante una actitud práctica de los aprendizajes obtenidos en el TCU en su cotidiano.

En este sentido, las personas encargadas se presentan bajo roles de personarecurso y asesor (Peplau, 1990). En primer lugar, para mostrar accesibilidad para el proceso de enseñanza-aprendizaje del estudiantado al establecer una horizontalidad basada en el respeto, y, en segundo lugar, para guiarlo en su sensibilización sobre la importancia del enfoque de las $\mathrm{HpV}$ para la prevención del consumo de drogas desde la acción en comunidad.

Esta relación se visualiza de ayuda como un sistema abierto de intercambio donde cada individuo que la conforma encuentra su beneficio al establecerse redes que se dupliquen una y otra vez en la comunidad de manera introspectiva, haciendo que los elementos de la tríada (docentes-estudiantes-comunidad) 
Revista Universidad en DiÁlogo • Vol. 10, N. ${ }^{\circ}$ 1, Enero-Junio, 2020 • 145-161

ISSN 2215-2849 • EISSN: 2215-4752

URL: http://www.revistas.una.ac.cr/index.php/dialogo/index CorReo ElECTRÓNICO: universidadendialogo@una.cr DOI: https://doi.org/10.15359/udre.10-1.9

conformadores de esta relación de ayuda se vean beneficiados (Neuman y Fawcett, 2002). Cada elemento toma un papel protagónico en el cumplimiento de las metas, empero, se sigue un proceso de mediación que garantice la responsabilidad social del trabajo de campo.

Siguiendo algunos conceptos de la filosofía de Benner (1984), apoyada en el modelo de adquisición de competencias de Dreyfus y Dreyfus (1980), los profesores responsable y colaborador, a partir de su formación académica y de su experiencia, se presentan en el estadio de competentes. Se enfocan en la gestión del tiempo y en la organización de las tareas que deben desarrollar las personas matriculadas para cumplir con su requisito de aprobación. Además, se preocupan por la transmisión de los conocimientos y de las habilidades de manera vivencial.

Por consiguiente, el estudiantado se presenta en el estadio principiante, ya que se enfrentará con experiencias nuevas. Adquiere los lineamientos a seguir fuera del contexto previo a presentarse a la comunidad en la actividad de inducción, permitiéndole avanzar de estadio a principiante avanzado. Posicionado desde esta perspectiva, demuestra su capacidad para dominar aspectos a los que se debe enfrentar en cada institución o comunidad. La experiencia continua en el desarrollo de sus tareas le permitirá convertirse en competente (Benner, 1984; Dreyfus y Deyfus, 1980).

El mismo proceso de mediación es facilitado en la comunidad con la esperanza de que pueda crecer en la adquisición de las $\mathrm{HpV}$ y la prevención en el consumo de drogas. A largo plazo, se espera que tanto el profesorado, el estudiantado y la comunidad logren recibirse como competentes en una dinámica relacional de equidad para que juntos, a partir del empoderamiento, evolucionen a los estadios eficiente y experto para construir una sociedad mejor (Benner, 1984; Dreyfus y Dreyfus, 1980; García et al., 2017).

Lo anterior permite visualizar la importancia de los roles de enseñanza por el interés creado en torno al tema central del TCU y la responsabilidad de transmisión de la información para lograr un impacto positivo en función del liderazgo otorgado a quienes participan directa e indirectamente en este proyecto (Peplau, 1990; García et al., 2017).

El enfoque de las $\mathrm{HpV}$ es una estrategia que se ha impulsado con la finalidad de favorecer el desarrollo personal de cada individuo a partir del entrenamiento de diferentes cualidades que permiten un mejor afrontamiento de los factores estresantes del ambiente y que se convierta en un eje transversal de su vida (Díaz 
URL: http://www.revistas.una.ac.cr/index.php/dialogo/index

CORREO ELECTRÓNICO: universidadendialogo@una.cr

DOI: https://doi.org/10.15359/udre.10-1.9

y Mejía, 2018). Su propuesta metodológica se fundamenta desde aristas teóricas como el constructivismo o el aprendizaje social, mediante la implementación de actividades educativas y lúdicas acordes a la etapa de crecimiento y de desarrollo de los grupos participantes (UCR, 2018; Mantilla, 2001).

Tanto la adolescencia como la adultez temprana se definen a partir de una construcción social que depende del escenario económico, la cultura y la sociedad donde se desenvuelvan. Sin embargo, se caracterizan por ser etapas del desarrollo humano que implican cambios físicos, cognoscitivos, emocionales y relacionales que las hacen únicas (Papalia, Feldman y Martorell, 2012).

La Comisión Interamericana para el Control del Abuso de Drogas (CICAD) señala que existe un uso temprano de drogas lícitas e ilícitas en la población adolescente de América, situación preocupante debido a los riesgos para la salud general de quienes las consumen y a los riesgos sociales que esto significa como deserción escolar, accidentes de tránsito, pobreza o delincuencia (Cicad, 2019). Costa Rica no está ajena a dicha realidad y, según datos presentados por el IAFA (2016), la adolescencia es la etapa donde inicia el uso de drogas, lo cual muestra mayor incidencia conforme se acerca a la edad adulta. Por esta razón, es que tiene como población meta a la adolescencia y a la juventud.

Por un lado, la adolescencia vive un momento crítico en el que busca construir su identidad a partir de la respuesta a las siguientes preguntas: ¿Quién soy? o ¿quién quiero llegar a ser? (Erikson, 2000). Esto permite pensar que ante tal búsqueda se expone a diferentes riesgos presentes en la situación social en la que se desenvuelve y que podría provocar una alteración de su salud. Algunos riesgos pueden estar relacionados con el comportamiento alimentario, la higiene, el sufrimiento psíquico, el sedentarismo, los comportamientos sexuales, la violencia y las lesiones no intencionales, y por supuesto el consumo de drogas lícitas e ilícitas (OMS, 2019).

Para contrarrestarlos, el TCU ofrece estrategias educativas para la adquisición de las HpV (Singla et al., 2019). La forma en que la población adolescente aprenda depende de la motivación que tenga. Larson y Wilson, citados por Papalia, Feldman y Martorell (2012), enfatizan en que la motivación intrínseca del adolescente favorece su deseo de aprender. No obstante, debe existir una duplicidad en las opciones que se les ofrece. Desde este punto de vista, busca crear espacios didácticos y atractivos que capturen a la población adolescente para que se cumplan sus objetivos y que trasciendan a su proyecto de vida (Corrales, Quijano y Góngora, 2017). 
Revista Universidad en DiÁlogo • Vol. 10, N. ${ }^{\circ}$ 1, Enero-Junio, 2020 • 145-161

ISSN 2215-2849 • EISSN: 2215-4752

URL: http://www.revistas.una.ac.cr/index.php/dialogo/index CorReo ElECTRÓNICO: universidadendialogo@una.cr

DOI: https://doi.org/10.15359/udre.10-1.9

Debido a lo anterior, el asesoramiento y la guía que reciba la adolescencia favorece su proceso de afrontamiento ante los diferentes estresores que determinan su estado de salud. El TC687 se posiciona como una actividad accesible para quienes estén interesados en recibir su propuesta y participar de ella (Corrales, Quijano y Góngora, 2017).

Por otro lado, en la juventud su capacidad de aprender se define como posformal, es decir, la experiencia misma es la que le ha permitido adquirir conocimientos. Se caracteriza por ser individual, flexible y emocional, ayudándole a los adultos a enfrentar el mundo (Piaget, 1999). En consideración, las HpV se fundan como herramientas que fortalecen tales características, por lo que su aprendizaje es favorable.

Además, desarrolla un proceso de recentramiento fundado en el cambio de la identidad construida de la adolescencia a una identidad adulta (Cicad, 2019). Este requiere acompañamiento para direccionar a los adultos en prácticas saludables que les permitan realizar el cambio en favor de su bienestar. El TC687 se posiciona como una oportunidad abierta para brindar el acompañamiento en las comunidades que lo soliciten.

También, se enfrentan en una etapa en la que deben construir relaciones más íntimas para satisfacer sus necesidades básicas. No lograr establecerlas provocaría que se aíslen, enfrentándose a riesgos como el consumo de drogas. Erikson (2000) enfatiza en que la resolución de esta etapa da por resultado la virtud del amor entendido como la capacidad de tener interés por los demás de manera solidaria. El amor por otros, pero, principalmente, por mí mismo, se podría fomentar en el interés por la adquisición de las habilidades cognitivas, emocionales y sociales que permitan controlar factores de riesgo como el consumo de drogas. El amor propio se convertiría en un estímulo que impulsa la adopción de conductas saludables que, en el caso de esta población, las adquiere en las actividades que el TCU promueve bajo la premisa de que nosotros mismos debemos ser lo que amamos (Bordignon, 2005).

\section{Conclusiones}

La metodología de sistematización de experiencias permite recuperar la experiencia del TCU para crear un registro que permita la construcción de nuevas evidencias.

En concordancia con la evidencia presentada en los ámbitos nacional e internacional, el TCU ha generado resultados inmediatos positivos en las poblaciones que 
ha intervenido desde el enfoque de $\mathrm{HpV}$ y ha demostrado la necesidad de que la práctica sea constante para que esos resultados se reflejen a lo largo de la vida.

La experiencia del TCU fortalece la responsabilidad social del estudiantado con las comunidades del país. Con el acercamiento a dichas poblaciones, identifican diferentes realidades que los sensibilizan a partir de la compasión por las personas con mayores necesidades, creando relaciones de ayuda que se traducen en la acción social.

El TC687 implementa una estrategia ganar-ganar. El beneficio es mutuo, tanto para el estudiantado que matricula como para las instituciones-comunidades donde se implementa el proyecto. El proceso de enseñanza-aprendizaje de las habilidades para la vida sigue la línea extrospectiva e introspectiva, logrando coherencia con los objetivos y las metas propuestas.

$\mathrm{Su}$ desarrollo depende de un compromiso diario, ya que la puesta en práctica no es sencilla. Ante esto, se requiere que la propuesta del TC687 se mantenga a largo plazo en las instituciones o las comunidades para alcanzar los resultados esperados. Mientras más capacitación exista, todas las personas adquirirán herramientas para enfrentar los retos de la vida en un sentido positivo, permitiéndoles tomar mejores decisiones respecto al consumo de drogas.

Si bien el TC687 cumple objetivos y metas definidas en la formulación del proyecto, durante la ejecución busca trascender la vida de todas las personas participantes en otras áreas que favorezcan su bienestar y calidad de vida, debido a que el enfoque de las $\mathrm{HpV}$ es aplicable en los retos cotidianos y no solo en la prevención del consumo de drogas.

\section{Referencias bibliográficas}

Badilla, D. y Salazar, D. (2019). Intervención de enfermería desde la teoría de relaciones interpersonales en enfermería, para el fomento de habilidades para la vida con niños y niñas que residen en Aldeas Infantiles SOS Limón, Costa Rica 2018. Informe Final de Práctica Dirigida para optar por el grado de Licenciatura de Enfermería.

Benner, P. (1984). From novice to expert: Excellence and power in clinical nursing practice. Menlo Park, CA: Addison-Wesley.

Bordignon, N. (2005). El desarrollo psicosocial de Eric Erikson. El diagrama epigénico del adulto. Revista Lasallista de Investigación, 2(2), 50-63. https://www.redalyc.org/pdf/695/69520210.pdf 
Revista Universidad en DiÁlogo • Vol. 10, N. ${ }^{\circ}$ 1, Enero-Junio, 2020 • 145-161

ISSN 2215-2849 • EISSN: 2215-4752

URL: http://www.revistas.una.ac.cr/index.php/dialogo/index CORREO ELECTRÓNICO: universidadendialogo@una.cr DOI: https://doi.org/10.15359/udre.10-1.9

Cicad (2019). Informe sobre el consumo de drogas en las Américas. Washington D.C.: OEA.

Consejo Universitario (1974). Estatuto Orgánico de la Universidad de Costa Rica. Alcance 52 a La Gaceta Oficial. 56, 1-46.

Corrales, A., Quijano, N. y Góngora, E. (2017). Empatía, comunicación asertiva y seguimiento de normas. Un programa para desarrollar habilidades para la vida. Enseñanza e Investigación en Psicología, 22(1), 5865. http://www.redalyc.org/articulo.oa?id=29251161005

Díaz, M. y Mejía, S. (2018). Desarrollo de habilidades para la vida en la prevención del consumo de sustancias psicoactivas: un enfoque crítico al modelo existente. El Ágora USB, 18(1), 203-210. http://dx.doi. org/10.21500/16578031.3450

Dreyfus, S. y Dreyfus, H. (1980). A five-stage model of the mental activities involved in directed skill acquisition. Berkeley, CA: University of California.

Erikson, E. (2000). El ciclo vital completado. Barcelona: Paidós Ibérica.

García, G., Givaduan, M., Ramírez, M., Vladez, E. y Pick, S. (2017). El cuidado de la salud como semilla para el desarrollo: experiencia de un programa basado en habilidades para la vida y reducción de barreras psicosociales. Acta de Investigación Psicológica, 7, 2647-2657. https:// dx.doi.org/10.1016/j.aipprr.2017.06.002

IAFA (2016). IV Encuesta Nacional sobre Consumo de Drogas en Población de Educación Secundaria. San José: IAFA.

Jara, O. (2012). La sistematización de experiencias. Práctica y teoría para otros mundos posibles. San José: Centro de Estudios y Publicaciones Alforja.

La Gaceta Universitaria (2018). Reglamento del Trabajo Comunal Universitario de la Universidad de Costa Rica. Alcance a La Gaceta Universitaria, 18, 1-8.

Mantilla, L. (2001). Habilidades para la vida. Una propuesta educativa para la promoción del desarrollo humano y la prevención de problemas psicosociales ( $2^{\mathrm{da}}$ ed.). Bogotá: Ministerio de Salud y Fe y Alegría. 
Murillo, L. y Ramírez, O. (2014). Programa de enfermería para el desarrollo de habilidades para la vida en los residentes del Hogar Crea de niñosadolescentes, Birrisito, 2014. Informe Final de Práctica Dirigida para optar por el grado de Licenciatura de Enfermería.

Neuman, M. y Fawcett. (2002). The Neuman Systems Model (4 ${ }^{\text {th }}$ ed.). New Jersey: Prentice Hall.

NIDA (2004). Cómo prevenir el abuso de drogas en los niños y adolescentes. Una guía con base cientifica para padres, educadores y líderes de la comunidad ( $2^{\mathrm{da}}$ ed.). Maryland: NIH.

OMS (25 de febrero, 2019). Encuesta Mundial de Salud a Escolares. Ginebra: OMS. Recuperado de https://www.who.int/ncds/surveillance/gshs/es/

OPS (2001). Enfoque de habilidades para la vida para un desarrollo saludable de niños y adolescentes. Washington D.C.: OPS.

Papalia, D., Feldman, R. y Martorell, G. (2012). Desarrollo humano. México, D. F.: McGraw-Hill.

Peplau, H. (1990). Relaciones interpersonales en enfermería. Un marco de referencia conceptual para la enfermería psicodinámica. Barcelona: Salvat Editores.

Piaget, J. (1999). La psicología de la inteligencia. Barcelona: Crítica.

Rogers, C. (2011). El proceso de convertirse en persona. Mi técnica terapéutica. Madrid: Paidós Contextos.

Singla, D. et al. (2019). Implementation and effectiveness of adolescent life skills programs in low and middle-income countries: a critical review and meta-analysis. Behavior Research and Therapy. https://doi. org/10.1016/j.brat.2019.04.010 